\title{
CONSULTORIAS ONLINE: UMA NOVA PERSPECTIVA NO TRABALHO DA ENFERMAGEM*
}

\author{
Magda Guimarães de Araujo Fariaํㅜㄹ Helena Maria Scherlowski Leal David², Sonia Acioli²
}

\begin{abstract}
RESUMO: Objetivou-se descrever a participação dos enfermeiros em teleconsultorias online em um programa de educação permanente à distância. Para tanto, utilizou-se a metodologia quantitativa, com desenho longitudinal e observacional, delineamento de pesquisa não experimental e caráter descritivo. Os dados foram obtidos entre os anos de 2009 e 2010 utilizando registros de perguntas e respostas de segunda opinião formativa, no banco de dados do referido Programa. Verificou-se que a maior parte das dúvidas dos enfermeiros cadastrados e usuários do Programa é pautada em dúvidas clínicas, seguidos de dúvidas sobre implementação de atividades educativas. Conclui-se pela viabilidade e importância desta atividade de educação permanente, em que pesem algumas contradições e dificuldades para o enfermeiro da atenção básica os quais se constituem em desafios a serem superados. DESCRITORES: Educação continuada; Educação à distância; Telenfermagem; Telessaúde.
\end{abstract}

\section{ONLINE CONSULTATIONS: A NEW PERSPECTIVE ON THE WORK OF NURSING}

ABSTRACT: The aim was to describe nurses' participation in on-line teleconsultations in a distance-learning continuous education program. Quantitative methodology was used to this end, with a longitudinal and observational design, a nonexperimental research outline, and a descriptive character. The data was obtained in the years 2009 and 2010, using records of questions and answers, from the above-mentioned program's database. It was ascertained that the majority of the doubts of the nurses registered and using the program is based on clinical aspects, followed by doubts about implementing educational activities. It is concluded that this continuous education activity is viable and important, in which there are some contradictions and difficulties for the primary care nurse - which constitute challenges to be overcome.

DESCRIPTORS: Continuous education; Distance learning; Telenursing; Telehealth.

\section{CONSULTORIASONLINE: UNA NUEVA PERSPECTIVA ENELTRABAJODE LA ENFERMERÍA}

RESUMEN: El objetivo del trabaljo fue describir la participación de enfermeros en teleconsultorías online en programa de educación permanente a distancia. Para eso, fue utilizada la metodología cuantitativa, con dibujo longitudinal y observacional, delineamento de investigación no experimental y carácter descriptivo. Los datos fueron obtenidos entre los años de 2009 y 2010, utilizando registros de preguntas y respuestas de segunda opinión formativa, en el banco de datos del programa mencionado. Se ha verificado que la mayor parte de las dudas de los enfermeros registrados y usuarios del programa es pautada en aspectos clínicos, seguidos de dudas sobre implementación de actividades educativas. Se concluye que es viable y muy importante esta atividade de educación permanente, en la cual hay algunas contradicciones y dificultades para el enfermero de la atención básica que se constituyen en desafíos a superar.

DESCRITORES: Educación continuada; Educación a distancia; Telenfermería; Telesalud.

\footnotetext{
*Artigo baseado na dissertação 'Telessaúde Brasil - Núcleo Rio de Janeiro: a educação permanente no trabalho de enfermeiros na atenção básica, apresentada ao Programa de Pós-Graduação em Enfermagem da Universidade do Estado do Rio de Janeiro, em 2010.

${ }^{1}$ Enfermeira. Mestre em Enfermagem. Doutoranda pelo Programa de Pós-Graduação em Enfermagem da Universidade Estadual do Rio de Janeiro - UERJ. Teleconsultora de Enfermagem do Telessaúde Brasil - Núcleo Rio de Janeiro. Professora substituta do Departamento de Saúde Pública da Faculdade de Enfermagem da UERJ. Bolsista CAPES.

${ }^{2}$ Enfermeira. Doutora em Saúde Pública. Professora da Faculdade de Enfermageme do Programa de Pós-Graduação em Enfermagem da UERJ.
}

Autor correspondente:

Magda Guimarães de Araujo Faria

Universidade Estadual do Rio de Janeiro

Rua Dr. Satamini, 280 - 20270-231 - Rio de Janeiro-RJ-Brasil

E-mail: mag_araujo@yahoo.com.br
Recebido: 26/10/2012

Aprovado: 22/04/2013

Cogitare Enferm. 2013 Abr/Jun; 18(2):274-9 


\section{INTRODUÇÃO}

O profissional de saúde passa sua trajetória profissional em um intenso e constante processo educativo e a inserção de novas tecnologias, novos procedimentos e novos saberes em seu cotidiano influenciam este processo.

Para o enfermeiro atuante na atenção básica, estratégias de educação permanente tornam-se indispensáveis para atualizar o profissional a respeito de conceitos e práticas. Além disso, os programas de educação permanente, nos quais os profissionais de enfermagem se inserem, possibilitam o desenvolvimento de habilidades específicas, tais quais a postura crítica, auto-avaliação e autogestão. Isto porque, numa concepção crítica, não há como separar a educação e o trabalho ${ }^{(1)}$. Dessa forma, pode-se observar que a educação a distância (EAD) é grande aliado na educação permanente dos profissionais de saúde, pois permite o acesso do profissional a um determinado assunto, ao mesmo tempo que o instiga a buscar maiores informações, utilizando a mesma temática ${ }^{(2)}$.

Nesta perspectiva, o Ministério da Saúde instituiu em 2007 o Programa Telessaúde Brasil, visando a oferta de educação permanente a distância para profissionais da atenção básica de saúde. O objetivo do Telessaúde Brasil é integrar as equipes de saúde da família das diversas regiões do país com os centros universitários de referência, melhorando, a qualidade dos serviços prestados em atenção primária, auxiliando a diminuir o custo de saúde através da qualificação profissional, redução de deslocamentos desnecessários e através do aumento de atividades de prevenção de doenças ${ }^{(3)}$.

No Estado do Rio de Janeiro, o projeto piloto foi criado para atender a demanda dos profissionais da Estratégia Saúde da Família (ESF) e conta com equipe especializada de diversas áreas de abrangência, tais como enfermeiros, fisioterapeutas, médicos, nutricionistas e odontólogos.

Baseado na experiência profissional e na necessidade existente entre profissionais da ESF em expor dúvidas do seu cotidiano, o Projeto Telessaúde Brasil possui uma ferramenta assíncrona voltada a essa demanda: a segunda opinião formativa. Através dela, o profissional tem a oportunidade de enviar uma dúvida para segunda opinião formativa, a qual será analisada por um especialista na área específica, denominado teleconsultor. É válido lembrar que todo esse processo ocorre em, no máximo 72 horas, com garantia de sigilo absoluto ${ }^{(4)}$.
A Universidade do Estado do Rio de Janeiro teve um importante papel em todo o processo de expansão do Projeto Telessaúde Brasil, uma vez que foi a instituição responsável pela implantação do Telessaúde Núcleo Rio de Janeiro (TSRJ). Além de disponibilizar o espaço físico para o Projeto, contribuiu com os recursos humanos, por meio da inserção de docentes como coordenadores e teleconsultores, organizados em áreas profissionais e temáticas. Atualmente, as Faculdades de Ciências Médicas, Enfermagem, Odontologia, Nutrição, dentre outras, possuem representantes no corpo do Projeto, além do desenvolvimento de atividades em áreas temáticas prioritárias para a Atenção Básica, como hanseníase e saúde do idoso.

O objetivo do presente trabalho é descrever e analisar a utilização da atividade de teleconsultoria para o processo de educação permanente dos enfermeiros dos municípios fluminenses que participam do Projeto.

\section{MÉTODO}

O presente estudo foi desenvolvido por meio de abordagem quantitativa com desenho longitudinal e observacional, delineamento de pesquisa não experimental e caráter descritivo.

A pesquisa se baseia em análise de registros em banco de dados do TSRJ e compreendeu a análise do registro de segunda opinião formativa. Este serviço, também chamado de teleconsultoria, foi proposto pelo Programa Telessaúde Brasil como forma de suprir uma necessidade identificada pelos propositores do Programa. Partiu-se da ideia de que muitos profissionais trabalham em áreas remotas e, devido a barreiras geográficas e temporais, nem sempre possuem apoio de outros profissionais para solucionar suas dúvidas técnicas. Esta necessidade seria então suprida por um sistema de teleconsultoria individualizado, pelos teleconsultores da equipe do Telessaúde.

As dúvidas são categorizadas no Programa da seguinte forma: abordagem comunitária, abordagem familiar, casos clínicos, dúvida em clínica geral, educação em saúde, planejamento ou gestão, processo de trabalho da equipe e outras dúvidas. O envolvimento da equipe de enfermagem com esse tipo de atividade teve início em maio de 2009 e em junho de 2010 (momento que marca o final da coleta de dados deste estudo), período que havia 99 registros de pedidos de segunda opinião formativa. 
Ressalta-se que a pesquisa foi baseada em banco de dados secundários, não se fazendo necessário o Termo de Consentimento Livre e Esclarecido. Porém, o resultado e toda análise conclusiva foi exposto para os enfermeiros cadastrados no TSRJ, através de teleconferência participativa. O projeto foi apreciado e aprovado pelo comitê de ética em pesquisa sob Parecer de n. 2552/2009 e também pela equipe do Telessaúde.

\section{RESULTADOS}

A partir dos 99 registros foram avaliadas as principais demandas de dúvidas, além de verificar o grau de satisfação. A distribuição dos pedidos de segunda opinião formativa está apresentada na tabela 1; os pedidos de segunda opinião formativa são classificados quanto ao tipo de dúvida (figura 1) e divididos por regiões e tipo de dúvida (tabela 2).

Tabela 1 - Pedidos de segunda opinião formativa do Programa Telessaúde Núcleo Rio de Janeiro. Rio de Janeiro, 2010

\begin{tabular}{cccccccccccccc}
\hline & Jan & Fev & Mar & Abri & Mai & Jun & Jul & Ago & Set & Out & Nov & Dez & Total \\
\hline 2009 & 0 & 0 & 0 & 0 & 11 & 14 & 20 & 9 & 20 & 10 & 2 & 0 & 86 \\
2010 & 1 & 1 & 3 & 2 & 3 & 3 & - & - & - & - & - & - & 13 \\
\hline
\end{tabular}

Tabela 2 - Quantitativo de pedidos de segunda opinião formativa do Programa Telessaúde Núcleo Rio de Janeiro de acordo com as temáticas e regiões do Estado. Rio de Janeiro, 2010

\begin{tabular}{|c|c|c|c|c|c|c|c|c|}
\hline Região & Centro-sul & Litorânea & $\begin{array}{l}\text { Médio- } \\
\text { Paraíba }\end{array}$ & Metropolitana & Noroeste & Norte & Serrana & Total \\
\hline $\begin{array}{l}\text { Abordagem } \\
\text { comunitária }\end{array}$ & 0 & 1 & 1 & 5 & 1 & 0 & 1 & 10 \\
\hline $\begin{array}{l}\text { Abordagem } \\
\text { familiar }\end{array}$ & 0 & 2 & 1 & 4 & 2 & 0 & 3 & 12 \\
\hline Clínica geral & 0 & 6 & 5 & 7 & 2 & 0 & 7 & 27 \\
\hline $\begin{array}{l}\text { Educação em } \\
\text { saúde }\end{array}$ & 0 & 2 & 5 & 11 & 0 & 0 & 1 & 19 \\
\hline $\begin{array}{l}\text { Planejamento } \\
\text { ou gestão }\end{array}$ & 0 & 4 & 2 & 2 & 0 & 0 & 0 & 8 \\
\hline $\begin{array}{l}\text { Processo de } \\
\text { trabalho }\end{array}$ & 0 & 2 & 1 & 4 & 1 & 1 & 0 & 9 \\
\hline Outras & 0 & 3 & 0 & 7 & 2 & 0 & 2 & 14 \\
\hline Total & 0 & 20 & 15 & 40 & 8 & 2 & 14 & 99 \\
\hline
\end{tabular}

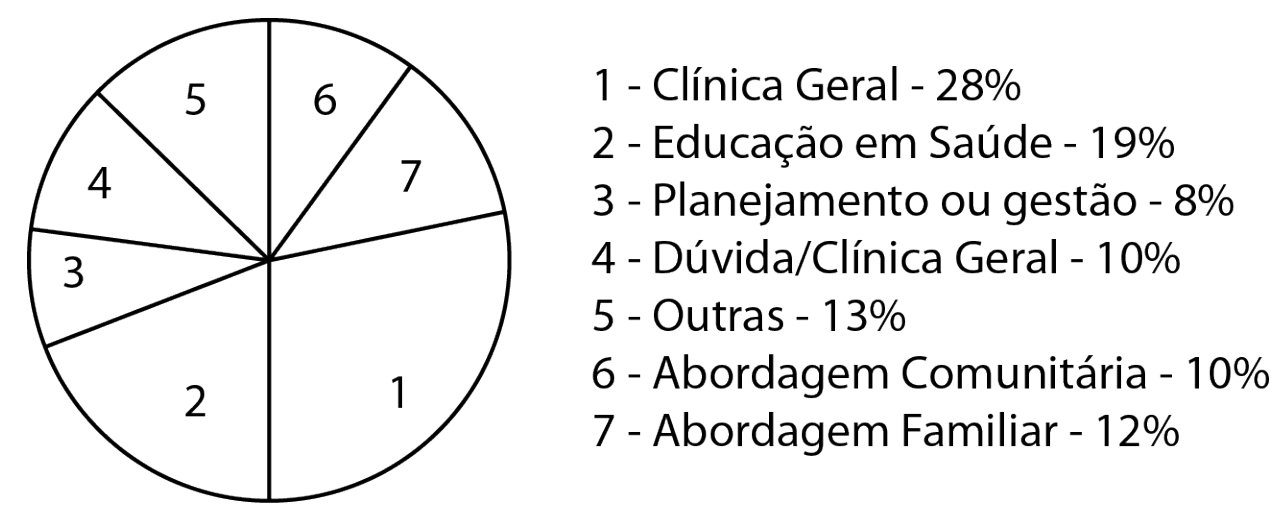

Figura 1 - Percentual de pedidos de segunda opinião formativa do Programa Telessaúde Núcleo Rio de Janeiro classificados por temáticas específicas - Rio de janeiro, 2010 


\section{DISCUSSÃO}

A análise dos dados aponta algumas modificações quanto ao padrão de pedidos de segunda opinião formativa, com aumento e concentração em junho, julho setembro e outubro de 2009. Atribuímos como precursor deste fato as oficinas presenciais realizadas em núcleos regionais, as quais contaram com a participação de profissionais do TSRJ e também de trabalhadores da atenção básica. Estas atividades ocorreram apenas em algumas áreas do Estado, foram realizadas quatro oficinas, nas regiões do Médio Paraíba, Serrana, Baixada Litorânea e também na região Metropolitana.

Os dados confirmam que, a partir do momento que o profissional de saúde tem contato direto com o teleconsultor do Projeto por meio de oficinas, a comunicação virtual posterior entre os mesmos ocorre de modo facilitado. A proposta da equipe em incentivar o uso do serviço de segunda opinião formativa é, sem dúvida, atendida em todas as oficinas presenciais. Mas, segundo os registros, muitos profissionais tendem a somente realizar o pedido de segunda opinião formativa nas oficinas presenciais, de modo que o serviço se torna subutilizado. Para ilustrarmos este fato, podemos citar a primeira atividade presencial ocorrida na região do Médio Paraíba, em maio do ano de 2009. Neste mesmo mês, foram registrados na base de dados do TSRJ, 11 teleconsultorias, correspondentes a todo o quantitativo do mesmo mês, porém nos meses seguintes, foram registrados apenas quatro pedidos de segunda opinião formativa.

O mesmo ocorreu na região Serrana no mês seguinte. Em junho de 2009, foram registrados 11 pedidos de segunda opinião formativa logo após a atividade presencial realizada na região. Assim como na região do Médio Paraíba, o número de pedidos deste serviço decaiu de modo que apenas em julho foram registrados novos pedidos da atividade. Outra região contemplada com a realização de atividades presenciais foi a Baixada Litorânea onde se observou que os pedidos de segunda opinião formativa continuaram nos meses seguintes, porém com demanda inferior.

No mês de setembro de 2009 a equipe de enfermagem do TSRJrealizou uma nova atividade presencial, desta vez contando com a participação dos profissionais da região Metropolitana. Ao contrário do que acontecia em outras regiões do Estado, a área citada já havia realizado pedidos de segunda opinião formativa antes da atividade presencial. Após esta, os pedidos decaíram, porém continuaram a surgir nos meses seguintes. Além disso, a região metropolitana foi a mais expressiva neste tipo de atividade no ano de 2010, sendo responsável por mais de $50 \%$ das solicitações deste serviço no presente ano.

Percebeu-se que nas regiões que receberam as atividades presenciais houve aumento significativo na demanda de segunda opinião formativa. Uma das principais hipóteses para este aumento é o fortalecimento do vínculo entre profissionais da ESF e teleconsultores do telessaúde. Esse vínculo não pode ser caracterizado apenas como um contato visual e sim pela escuta dos profissionais em encontros presenciais. O papel desempenhado pelo professor em EAD é de ser orientador dos estudos, porém este deverá fornecer também, apoio psicossocial ${ }^{(5)}$. O professor em EAD amplia sua área de atuação, porém não o isenta da interação professor-aluno. O docente deve procurar maneiras criativas para o estabelecimento do vínculo com o aluno, ciente de que sua ação profissional não pode ser substituída pela tecnologia ${ }^{(6)}$.

Cabe ao enfermeiro da unidade básica de saúde ações como atividades assistenciais voltadas para o cuidado, consultas de enfermagem e a realização de curativos; as atividades administrativas voltadas para o cuidado, como a supervisão dos agentes comunitários de saúde, as reuniões de equipe e o acolhimento; as atividades educativas voltadas para o cuidado, como as ações educativas voltadas para a população e a capacitação dos agentes comunitários ${ }^{(7)}$. De acordo com a análise das temáticas específicas, verificamos que mesmo com todas as atribuições pertinentes ao enfermeiro atuante na ESF, as dúvidas em clínica geral de agentes comunitários de saúde ainda são as mais constantes no cotidiano deste profissional.

Para análise quanto aos possíveis determinantes deste fato, podemos levantar três hipóteses. A primeira é que esta busca por temas clínicos condiz com a realidade do cotidiano das práticas deste trabalhador. Na grande maioria das unidades de Atenção Básica há alta demanda de conhecimento técnico no que se refere a procedimentos oriundos da prática da enfermagem, tais como curativos, pré-natal, acompanhamento aos doentes crônicos, dentre outras.

A segunda hipótese da análise do alto percentual de dúvidas em clínica geral situa-se em torno da herança do modelo biomédico de saúde, ainda presente no cotidiano das práticas. O modelo biomédico possui como característica a assistência a saúde focada no ato prescritivo, observando a doença de maneira objetiva e, excluindo aspectos subjetivos que acompanham 
qualquer enfermidade ${ }^{(8)}$.

A terceira hipótese levantada tem origem na não adesão dos profissionais em outra categoria de dúvida, chamada caso clínico, que foi criada originalmente para troca de informações de um paciente específico. Contudo, foi observado que os enfermeiros não utilizam este recurso devido os requisitos éticos relativos a dados e autorização dos pacientes. A não utilização deste tipo de dúvida permitiu o aumento de outra categoria: as dúvidas em clínica geral, onde o enfermeiro expõe apenas o quadro de saúde do paciente. Vale ressaltar que, desta maneira, o profissional consegue trocar informações com o teleconsultor, mas fica restrito a utilização de imagens do paciente como fotos de úlceras, por exemplo.

Ainda, na análise das demandas do serviço de segunda opinião formativa verificamos alto índice da demanda de educação em saúde, este facilmente explicado quando observamos uma das principais atribuições do enfermeiro junto à sua equipe multidisciplinar. Ele é responsável pela educação permanente da sua equipe, em especial os técnicos, auxiliares de enfermagem e agentes comunitários de saúde ${ }^{(9)}$.

Além de representar uma parte do processo de trabalho do enfermeiro atuante na ESF, a educação da equipe, assim como a educação em saúde, se faz uma importante ação para a promoção de saúde $^{(10)}$. Apesar da educação em saúde ser uma atribuição do enfermeiro da unidade básica de saúde, muitos desses ainda possuem dúvidas de como colocar esta ação em prática. A mobilização da equipe e a utilização de recursos para a prática da educação permanente compõem as principais demandas de dúvidas dentro da categoria.

Quanto a demandas de abordagens comunitária e familiar, verificamos que apesar do baixo índice de dúvidas, elas correspondem principalmente a dificuldades em relação a estabelecimento de vínculos com a comunidade e também em relação a membros de um determinado lar. Como exemplo deste tipo de demanda, recebemos muitas questões em relação à abordagem de famílias com problemas do tipo alcoolismo, doenças infecciosas e gestação precoce.

Todos os profissionais atuantes na ESF devem conhecer a realidade das casas onde atuam, além de junto aos membros da comunidade desenvolver ações de promoção de saúde e prevenção de doenças ${ }^{(11-12)}$. Para tanto, o enfermeiro deve estar apto a desenvolver diálogo com a comunidade e também as famílias assistidas.
Quando falamos de processo de trabalho na ESF, devemos lembrar que o modelo rompe com o sistema tradicional de cuidar em saúde. Podemos citar como parte integrante do processo de trabalho na Estratégia: o atendimento domiciliar, discussão de casos, reuniões de planejamento e também reuniões com a comunidade. O profissional deve incorporar ao processo de trabalho, o estabelecimento de vínculos com a comunidade e com as famílias assistidas ${ }^{(13)}$.

As dúvidas ao processo de trabalho são relacionadas a aspectos práticos do cotidiano do profissional em relação a tarefas burocráticas, assim como a demanda de questões relacionadas ao planejamento e gestão. Para exemplificar este tipo de demanda, podemos citar as dúvidas em relação à progressão do processo de trabalho de enfermagem na ESF, além do planejamento de reuniões com a equipe. É interessante analisar que, apesar da relação da demanda de segunda opinião formativa e as atividades presenciais, notamos grande heterogeneidade em relação às regiões político-administrativas do Estado do Rio de Janeiro.

Podemos notar ainda que, em relação às demandas dos enfermeiros, há grande diferença entre o trabalho prescrito e o real. O trabalho prescrito do enfermeiro seria relativo às atribuições já expostas nesta pesquisa e, o trabalho real é justamente as atividades realizadas pelos mesmos sujeitos ${ }^{(14)}$. Para exemplificarmos, podemos lembrar uma das principais atividades realizadas pelos enfermeiros na atenção básica de saúde: a visita domiciliar. Em nossa pesquisa, não foi registrada segunda opinião formativa que abordasse o tema, o que nos sugere que há distância entre o trabalho prescrito do enfermeiro e seu trabalho real.

\section{CONCLUSÃO}

A EAD em saúde privilegia, essencialmente, profissionais que buscam a autogestão do aprendizado, configurando um complementador ao processo educativo presencial e contínuo. Além disso, esta modalidade não exclui as demais formas de busca por conhecimento.

A busca por atividades online traduz a necessidade de inovação nos meios educativos para profissionais de saúde. Entretanto, encontros presenciais com a população-alvo ainda são indispensáveis para o fortalecimento de vínculos entre tutores e alunos, o que os torna essenciais para a continuidade das atividades. 


\section{REFERÊNCIAS}

1. Oliveira MAN. Educação à distância como estratégia para educação permanente em saúde: possibilidades e desafios. Rev Bras Enferm. 2007;60(5):585-9.

2. Faria MGA, David HMSL. Enfermagem e Educação permanente à distância: O exemplo do Projeto Telessaúde Brasil Núcleo Rio de Janeiro. Cogitare enferm. 2010;15(4):667-73.

3. Ministério da Saúde (BR). Programa Nacional de Telessaúde: sobre o programa. Brasília: Ministério da Saúde; 2009 [Internet] [acesso em 26 ago 2012]. Disponível: http://www.telessaudebrasil.org.br/php/ level.php?lang=pt\&component=42\&item $=1$

4. Ministério da Saúde (BR). Projeto Telessaúde Brasil Núcleo Rio de Janeiro. Rio de Janeiro: UERJ; 2008 [Internet] [acesso em 26 ago 2012]. Disponível: http:// www.telessauderj.uerj.br/nucleo_rj.php

5. Belloni ML. Educação a Distância. $5^{\mathrm{a}}$ ed. Campinas: Autores Associados; 2009.

6. Kenski VM. Educação e tecnologias, o novo ritmo da informação. $4^{\mathrm{a}}$ ed. Campinas: Papirus; 2008.

7. Kebian LAV. As práticas de saúde do enfermeiro e do agente comunitário de saúde na visita domiciliar da ESF [dissertação]. Rio de Janeiro (RJ): Universidade do Estado do Rio de Janeiro; 2010.

8. Mitre SM, Andrade EIG, Cotta RMM. Avanços e desafios do acolhimento na operacionalização e qualificação do Sistema Único de Saúde na atenção primária: um resgate da produção bibliográfica do Brasil. Ciênc. saúde colet. 2012;17(8):2071-85.

9. Villas Boas LMFM, Araujo MBS, Timóteo RPS. A prática gerencial do enfermeiro no PSF na perspectiva de sua ação pedagógica educativa: uma breve reflexão. Ciênc. saúde colet. 2008;13(4):1355-60.

10. David HMSL, Acioli S. Mudanças na formação e no trabalho de enfermagem: uma perspectiva da educação popular e de saúde. Rev Bras Enferm. 2010;63(1):127-31.

11. Oliveira RL, Santos MEA. Educação em saúde na Estratégia de Saúde da Família: conhecimentos e práticas do enfermeiro. Revista Enfermagem Integrada. 2011;4(2):833-44

12. Lopes MCL, Marcon SS. Assistência à família na atenção básica: facilidades e dificuldades enfrentadas pelos profissionais de saúde. Acta Scientiarum. Biological Sciences. 2012;34(1):85-93.

13. Gomes ALC, Sá LD. As concepções de vínculo e a relação com o controle da tuberculose. Rev Esc Enferm USP. 2009;43(2):365-72.

14.

14. Chaves ACC, Silva VLA. Processo de trabalho e produção do cuidado na Estratégia de Saúde da Família: a realidade das enfermeiras. Rev. bras. cienc. saude. 2011;15(3):249-64. 